\title{
Influence of device to device interconnection elements on the system behavior and stability
}

\author{
IS Amiri ${ }^{1}$, Ahmed Nabih Zaki Rashed ${ }^{2}$, P. Yupapin ${ }^{3}$ \\ ${ }^{1,3}$ Computational Optics Research Group, Advanced Institute of Materials Science, Ton Duc Thang University, \\ Ho Chi Minh City, Vietnam \\ ${ }^{1,3}$ Faculty of Applied Sciences, Ton Duc Thang University, Ho Chi Minh City, Vietnam \\ ${ }^{2}$ Electronics and Electrical Communications Engineering Department Faculty of Electronic Engineering, \\ Menoufia University, Egypt
}

\begin{tabular}{l} 
Article Info \\
\hline Article history: \\
Received Aug 13, 2019 \\
Revised Nov 15, 2019 \\
Accepted Nov 29, 2019 \\
\hline
\end{tabular}

\section{Keywords:}

Focal length

Parallel crystal

Thin lens

Titled plane

\begin{abstract}
This work has presented the interaction between tangential/sagittal cylindrical thin lens in the titled plane parallel crystal. Stability criterion parameters are measured under the control of curvature radius of a spherical mirror, the thickness of the tilted plane crystal, the refractive index of tilted plane crystal, the thickness for a plate of matter and phase angle of the sagittal cylindrical thin lens. Beam radius waist is plotted against the focal length of the tangential cylindrical thin lens. Focal length for both thin lens in resonator crystal is optimized to upgrade the resonator system operation efficiency.
\end{abstract}

\section{Corresponding Author:}

IS Amiri,

Ton Duc Thang University,

Ho Chi Minh City, Vietnam.

E-mail: irajsadeghamiri@tdtu.edu.vn

\section{INTRODUCTION}

There are many types of thin lens that are namely tangential cylindrical thin lens, sagittal cylindrical thin lens and V-shaped thin lens [1-4]. There are many elements in the titled plane crystal resonators that are namely flat mirror, spherical mirror with curvature radius and phase angle variations [5-6]. The resonator crystal titled lane includes the first and second focal thin lens with the thickness, refractive index, and focal lengths. The resonators systems are divided by the space lengths suitable to make the resonator system is more stability and strength beam waist [7-10]. Many resonators system have many elements such as inductors, capacitors, and resistors. These components varied in its values according to achieve the resonator system in high-speed operation [11-13]. Resistors are varied in its values from $10 \Omega$ to $100 \mathrm{~K} \Omega$, capacitors also are varied from $1 \mathrm{mf}$ to $100 \mathrm{pf}$ and the inductors are varied from $1 \mathrm{mH}$ to $100 \mathrm{pH}$. The choice of these components values is dependent on the resonator system itself to achieve low delay time such as rise time and fall time. Some of the resonator components elements are used in high-speed systems [14-17]. CMOS technology, bipolar technology, NMOS plus PMOS technologies are employed in resonator systems [9]. The delay time, fall time and rise time are still the main propagation problems in resonator circuits. The maximum gain achieved is the major interesting criterion in high-speed resonator system [18-22]. 


\section{MODEL DESCRIPTION AND RESEARCH METHOD}

Figure 1 shows the basic simulation model for this study. M1 is the first element which represents the flat mirror. L1 is the second element which is represented by the space length and is taken a value of 100 $\mathrm{mm} . \mathrm{M} 2$ is the third item represents the spherical mirror with a curvature radius $\mathrm{R}$ and a phase angle alpha. The curvature radius of the mirror is positive for the concave mirror and is negative for a convex mirror. $\mathrm{Cr} 1$ is the plate of matter with a thickness of $15 \mathrm{~mm}$ and the refractive index of unity. L2 is the space length in $\mathrm{mm}$ units. F1 is the six elements in the resonator system which is re[resented tangential cylindrical thin lens in the air with focal length in $\mathrm{mm}$ units. The focal length of the lens is positive for collecting lens and negative for a diverging lens.

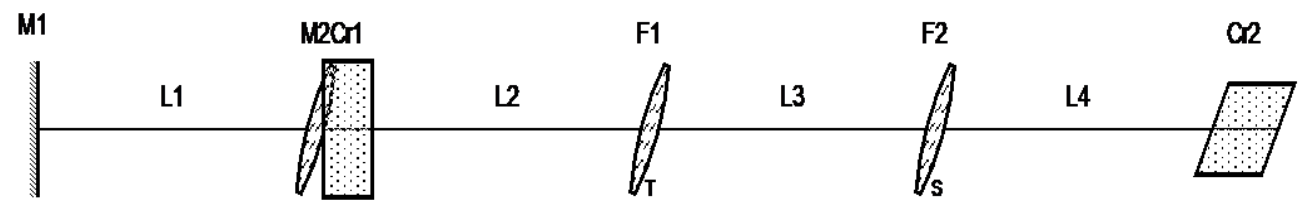

Figure 1. A basic simulation model for this work

L3 is the seven elements in the resonator system which is called as the space length. F2 is the sagittal cylindrical thin lens in the air with focal length in mm units and phase angle of alpha in degree. L4 is the space length between the lens and the Brewster crystal. L1 is the space length between the flat mirror and the spherical mirror. L2 is the space length between tangential/ sagittal cylindrical thin lens. Cr2 is the titled plane parallel crystal in the air with the thickness length in mm units and phase angle in degree and the refractive index of unity.

\section{PERFORMANCE ANALYSIS WITH DISCUSSIONS}

The simulation model is analyzed to show the interaction between tangential/sagittal cylindrical thin lens in the titled plane parallel crystal. The thickness of the plate of the matter is plotted against the curvature radius of the spherical mirror to measure the stability criterion. The curvature radius of the spherical mirror is plotted against thickness of titled plane parallel crystal to measure the stability parameter criterion. Beam radius variations versus is plotted against the focal length of the tangential cylindrical thin lens to measure beam waist accuracy. The focal length of tangential cylindrical thin lens variations is plotted versus refractive index of the titled plane crystal. Figure 2 indicates the relation between the stability criterion based thickness of the plate of matter against the curvature radius of the spherical mirror. The stability criterion based thickness of the plate of matter increases with increasing the curvature radius of the spherical mirror in both $\mathrm{S}$ and $\mathrm{T}$ planes. $\mathrm{T}$ plane has presented more stability than $\mathrm{S}$ plane up to $145 \mathrm{~mm}$ curvature radius. After 145 $\mathrm{mm}$ curvature radius, the $\mathrm{S}$ plane has outlined better stability than T plane. Figure 3 shows the basic relation between the basic stability criterion based curvature radius of spherical mirror variations versus thickness of the titled parallel crystal. Stability criterion concept based curvature radius of the spherical mirror is almost constant along the variations of the thickness of the titled parallel crystal. $T$ plane has outlined better performance in resonator system stability. Figure 4 review the beam radius variations versus tangential cylindrical thin lens variations. Beam radius waist increase with increasing thin focal length in $\mathrm{T}$ plane only. There is no component in $\mathrm{S}$ plane for beam radius waist variations. Beam radius variations from $100 \mathrm{~mm}$ to $250 \mathrm{~mm}$ with the variation values from the focal length of the lens from $40 \mathrm{~mm}$ to $60 \mathrm{~mm}$.

Figure 5 has outlined the stability factor based on focal length variations of refractive index variations of titled plane crystal in $\mathrm{T}$ plane. The stability factor is almost constant in the $\mathrm{S}$ plane. The stability factor decreases completely with increasing refractive index of titled plane crystal. Figure 6 indicates the stability criterion based focal length variations against the thickness of matter plate variations. Stability factor coefficient based tangential cylindrical thin lens increases with increasing thickness of plate matter. The stability is better through the values of $1 \mathrm{~mm}$ to $7.5 \mathrm{~mm}$ thickness of matter plate. But the stability criterion is dropped through the values of $7.5 \mathrm{~mm}$ up to $15 \mathrm{~mm}$ thickness of matter plate. The stability criterion variations against the phase angle of sagittal cylindrical thin lens variations are shown in Figure 7 . the stability parameter coefficient criterion decreases in both $\mathrm{S}$ and $\mathrm{T}$ planes. The stability criterion is reduced through the phase angle from 1 degree to 10 degrees. 


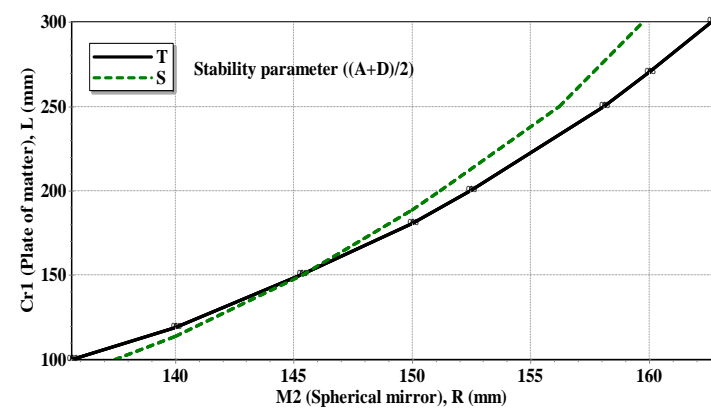

Figure 2. Stability criterion based thickness of the plate of matter against curvature radius of the spherical mirror

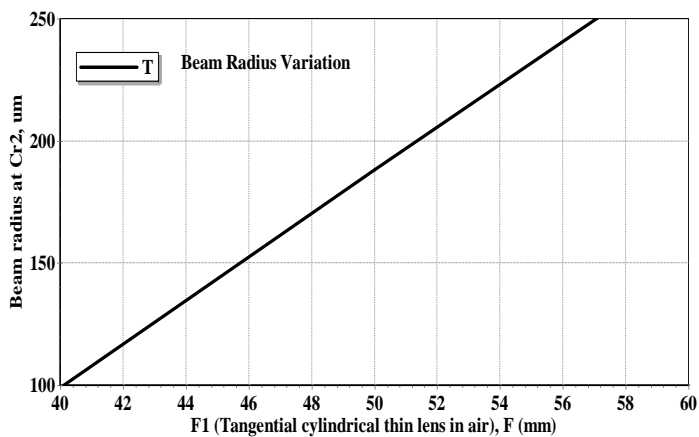

Figure 4. Beam radius variations versus tangential cylindrical thin lens variations

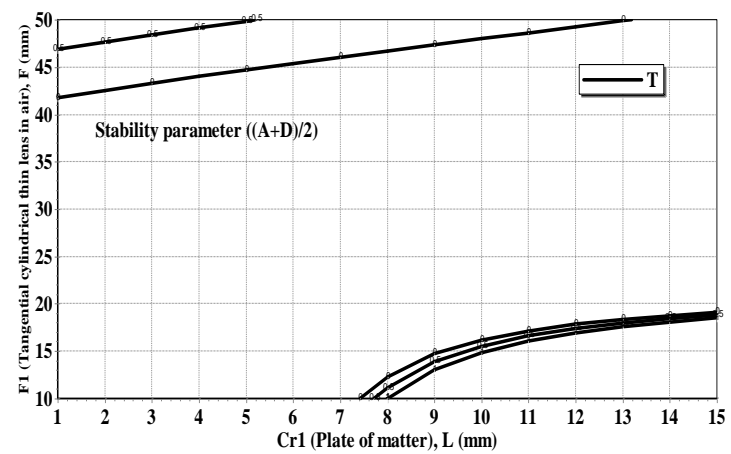

Figure 6. Variations of stability criterion based focal length versus thickness of matter plate variations

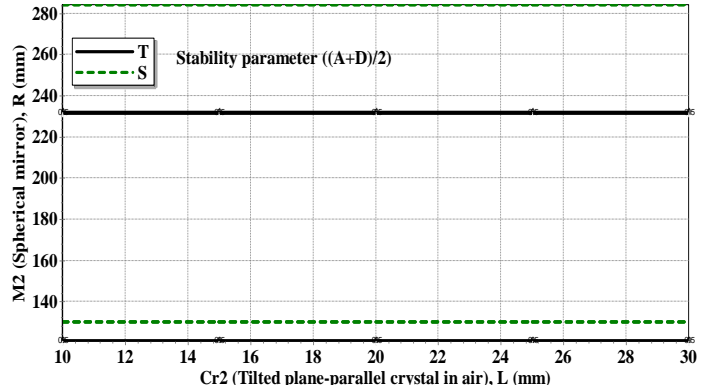

Figure 3. Stability criterion based curvature radius of spherical mirror variations versus thickness of the titled parallel crystal

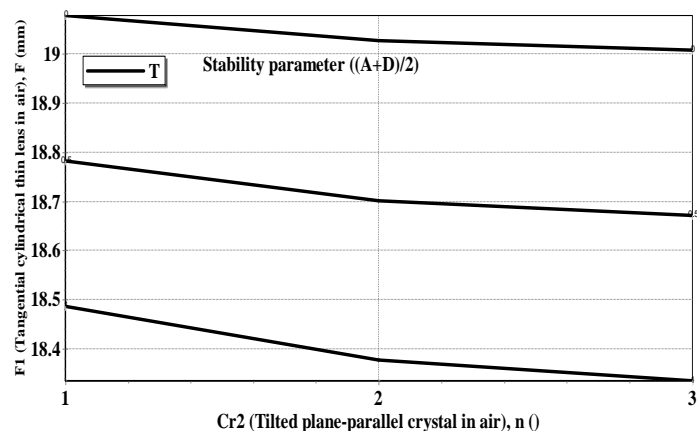

Figure 5. Focal length variations of refractive index variations of titled plane crystal

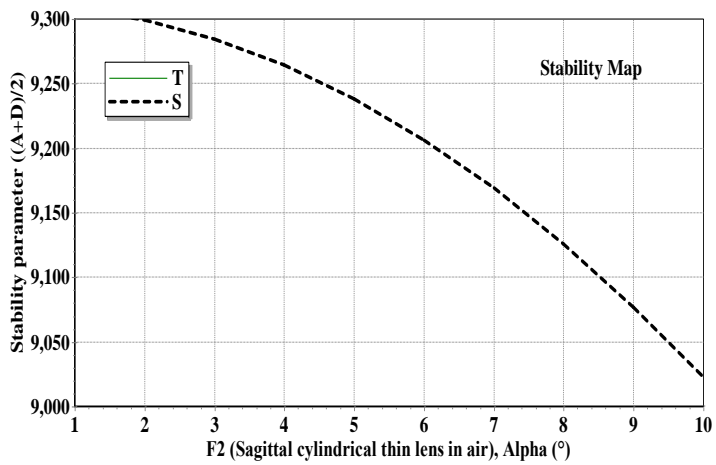

Figure 7. Stability criterion variations in relation to the phase angle of the sagittal cylindrical thin lens

The Stability criterion map parameter coefficient against the focal lens of tangential cylindrical thin lens variations is shown in Figure 8. The stability criterion based focal length of the thin lens in both T plane and $\mathrm{S}$ plane. The stability coefficient factor is dropped through focal length ranges from $1 \mathrm{~mm}$ to $6 \mathrm{~mm}$ and the stability is dropped badly through the range of $6 \mathrm{~mm}$ up to $10 \mathrm{~mm}$. Figure 9 outlines the relationship between the stability coefficient parameter against variations of the space length between system resonator elements. The more space length the larger the stability criterion coefficient in both $\mathrm{S}$ and $\mathrm{T}$ planes. The stability criterion is better performance in $\mathrm{S}$ plane than $\mathrm{T}$ plane. The intermode beat frequency of the resonator system is equal to $461.219 \mathrm{MHz}$ and total cavity resonator length is estimated as $100(\mathrm{~L} 1)+15(\mathrm{Cr} 1)$ $+100(\mathrm{~L} 2)+30(\mathrm{~L} 3)+50(\mathrm{~L} 4)+30(\mathrm{Cr} 2)=325 \mathrm{~mm}$. 


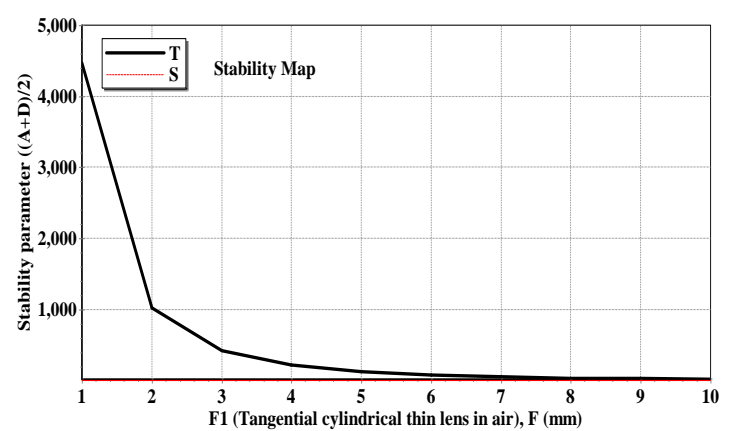

Figure 8. Stability criterion map against the focal lens of tangential cylindrical thin lens variations

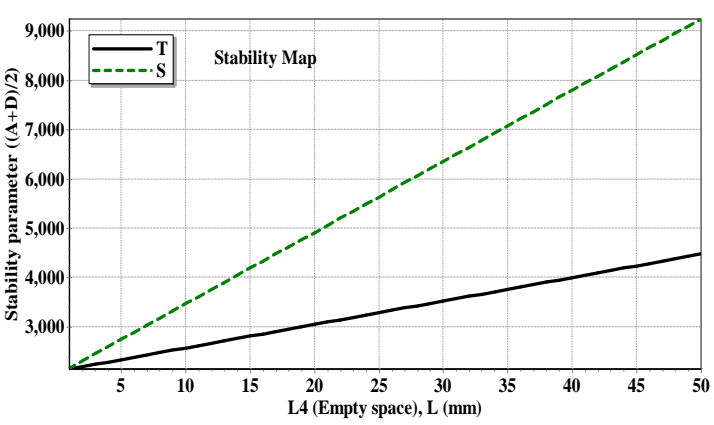

Figure 9. Stability criterion versus the space length between resonator elements

\section{CONCLUSION}

Beam radius waist and the stability criterion coefficient parameters are deeply studied. The higher the space length between resonator elements the higher the stability criterion and the higher the beam radius waist. The lower the focal thin lens the larger the stability parameter and beam waist strength. The lower the phase angle of thin lens the higher stability criterion and consequently the beam strength waist. The beat resonance frequency is reached to $461.219 \mathrm{MHz}$. the estimated total cavity resonator length is reached to 325 $\mathrm{mm}$. Therefore, the phase angle and focal length of thin lens should be optimized in order to achieve highspeed resonator system efficiency.

\section{REFERENCES}

[1] M. V. Raghavendra, et al., "Estimation of Optical Link Length for Multi Haul Applications," International Journal of Engineering Science and Technology, vol. 2, no. 6, pp. 1485-1491, 2010.

[2] S.-C. Wang, et al., "Optically Pumped GaN Based Vertical Cavity Surface Emitting Lasers: Technology and Characteristics," Jpn. J. Appl. Phys., vol. 46, pp. 5397-5407, 2007.

[3] T.-C. Lu, et al., "CW Lasing of Current Injection Blue GaN Based Vertical Cavity Surface Emitting Laser," Appl. Phys. Lett., vol. 92, pp. 1-3, 2008.

[4] Christopher J. Stapels, et al., "CMOS Based Avalanche Photodiodes for Direct Particle Detection," Nuclear Instruments and Methods in Physics Research A, vol. 579, no. 1, pp. 94-98, Aug. 2007.

[5] Pulvirenti, et al., "Characterization of Avalanche Photodiodes (APDs) for the Electromagnetic Calorimeter in the ALICE Experiment," Nuclear Instruments and Methods in Physics Research A, vol. 596, no. 1, pp. 122-125, Oct. 2008.

[6] Abd El-Naser A. Mohammed, et al., "Transient behavior and transmission bit rates analysis of optoelectronic integrated devices laser diode (LD) and light emitting diode (LED) under amplification and ionizing irradiation environments," Journal of Electrical and Electronics Engineering Research, vol. 3, no. 7, pp. 121-133, 2011.

[7] T. Lima, et al., "A Receiver Model for Optical Fiber Communication Systems With Arbitrarily Polarized Noise," Journal of Lightwave Technology, vol. 23, no. 3, pp. 1478-1490, Mar 2005.

[8] S. Norimatsu and M. Maruoka, "Accurate Q-factor Estimation of Optically Amplified Systems in the Presence of Waveform Distortions," Journal of Lightwave Technology, vol. 20, no. 1, pp. 19-27, Jan 2002.

[9] S. M. Jahangir Alam, et al., "Bit Error Rate Optimization in Fiber Optic Communications," International Journal of Machine Learning and Computing, vol. 1, no. 5, pp. 435-440, Dec 2011.

[10] S. M. J. Alam, et al., "Improvement of Bit Error Rate in Fiber Optic Communications," International Journal of Future Computer and Communication, vol. 3, no. 4, pp. 281-286, Aug 2014.

[11] Ahmed Nabih Zaki Rashed, and Mohamed A. Metwae'e, "Optical Filters Dimensions and Thermal Operation Conditions Impact on Its Transmission Considerations in Near Infrared (NIR) Optical Spectrum Transmission Region," Optoelectronics and Advanced Materials Journal-Rapid Communications, vol. 8, no. 3-4, pp. 175-184, March-April 2014.

[12] Taya, et al., "Design and Analysis of Low Power Universal Line Encoder \& Decoder," Microelectronics and Solid State Electronics Journal, vol. 5, no. 1, pp. 7-13, 2016.

[13] C.T. Manimegalai and S. G. Kalimuthu, "Underwater Optical Channel Analysis Using DPIM \& PPM," European Journal of Applied Sciences, vol. 9, no. 3 pp.154-162, 2017.

[14] Z. Kornain, et al., "The Simulation of Indoor Service Range Prediction of Wireless Radio Access Point for Radio over Fiber System," IACSIT International Journal of Engineering and Technology, vol. 5, no. 1, pp. 136-140, Feb 2013.

[15] Ahmed Nabih Zaki Rashed, "High Performance Photonic Devices For Multiplexing/Demultiplexing applications in Multi Band Operating Regions," Journal of Computational and Theoretical Nanoscience, vol. 9, no. 4, pp. 522-531, April 2012. 
[16] E. Feltin, et al., "Blue Lasing at Room Temperature in an Optically Pumped Lattice Matched AlInN/GaN VCSEL Structure," Electron. Lett., vol. 43, pp. 924-926, 2007.

[17] M. C. Shih and C. S. Chen, "A Semiconductor Soliton Cavity Laser Diode and its Output Characteristics," Japanese Journal of Applied Physics, vol. 50, no. 4, pp. 17-22, 2011.

[18] C.-C. Kao, et al., "The Lasing Characteristics of GaN Based Vertical Cavity Surface Emitting Laser With AlN$\mathrm{GaN}$ and $\mathrm{Ta}_{2} \mathrm{O}_{5}-\mathrm{SiO}_{2}$ Distributed Bragg Reflectors," IEEE Photon. Technol. Lett., vol. 18, no. 7, pp. 877-879, 2006.

[19] J.-T. Chu, et al., "Room-Temperature Operation of Optically Pumped Blue Violet GaN Based Vertical Cavity Surface Emitting Lasers Fabricated by Laser Lift Off," Jpn. J. Appl. Phys, vol. 45, pp. 2556-2560, 2006.

[20] J.-T. Chu, et al., "Emission Characteristics of Optically Pumped GaN Based Vertical Cavity Surface-Emitting Lasers," Appl. Phys. Lett., vol. 89, pp. 1-3, 2006.

[21] IS Amiri, Ahmed Nabih Zaki Rashed, "Simulative study of simple ring resonator-based brewster plate for power system operation stability," Indonesian Journal of Electrical Engineering and Computer Science (IJEECS), vol. 16, no. 2, pp. 1070-1076, November 2019, DOI: 10.11591/ijeecs.v16.i2.pp1070-1076.

[22] IS Amiri, Ahmed Nabih Zaki Rashed, "Numerical investigation of V shaped three elements resonator for optical closed loop system," Indonesian Journal of Electrical Engineering and Computer Science (IJEECS), vol. 16, no. 3, pp. 1392-1397, December 2019, DOI: 10.11591/ijeecs.v16.i3.pp1392-1397.

\section{BIOGRAPHIES OF AUTHORS}
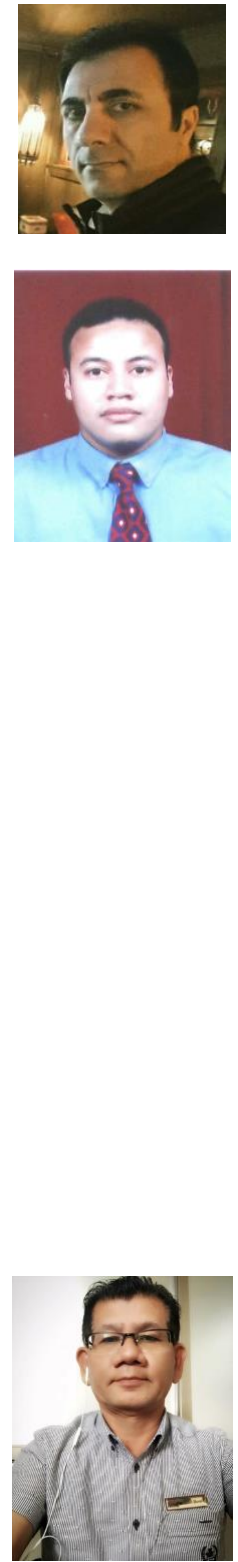

Dr. IS Amiri has been doing research on several topics such as the optical soliton communications, laser physics, fiber lasers, fiber grating, electro-optical modulators, nanofabrications, semiconductor design and modelling, Lumerical modelling, plasmonics photonics devices, nonlinear fiber optics, optoelectronics devices using 2D materials, semiconductor waveguide design and fabrications, photolithography fabrications, E Beam lithography, quantum cryptography and nanotechnology engineering.

Assoc. Prof. Ahmed Nabih Zaki Rashed was born in Menouf city, Menoufia State, Egypt country in 23 July, 1976. Received the B.Sc., M.Sc., and Ph.D. scientific degrees in the Electronics and Electrical Communications Engineering Department from Faculty of Electronic Engineering, Menoufia University in 1999, 2005, and 2010 respectively. Currently, his job carrier is a scientific lecturer in Electronics and Electrical Communications Engineering Department, Faculty of Electronic Engineering, Menoufia university, Menouf. Postal Menouf city code: 32951, EGYPT. His scientific master science thesis has focused on polymer fibers in optical access communication systems. Moreover his scientific $\mathrm{Ph}$. D. thesis has focused on recent applications in linear or nonlinear passive or active in optical networks. His interesting research mainly focuses on transmission capacity, a data rate product and long transmission distances of passive and active optical communication networks, wireless communication, radio over fiber communication systems, and optical network security and management.

He has published many high scientific research papers in high quality and technical international journals in the field of advanced communication systems, optoelectronic devices, and passive optical access communication networks. His areas of interest and experience in optical communication systems, advanced optical communication networks, wireless optical access networks, analog communication systems, optical filters and Sensors. As well as he is editorial board member in high academic scientific International research Journals. Moreover he is a reviewer member in high impact scientific research international journals in the field of electronics, electrical communication systems, optoelectronics, information technology and advanced optical communication systems and networks. His personal electronic mail ID (Email:ahmed_733@yahoo.com). His published paper under the title "High reliability optical interconnections for short range applications in high performance optical communication systems" in Optics and Laser Technology, Elsevier Publisher has achieved most popular download articles in 2013.

P. Yupapin received the Ph.D. degree in electrical engineering from the City, University of London, UK in 1993. He is currently the full Professor in the Computational Optics Research Group, Advanced Institute of Materials Science and member in the Faculty of Applied Sciences, Ton Duc Thang University, Ho Chi Minh City, Vietnam. His current researches of interest are nano-devices and circuits, microring resonator, soliton communication, optical motor, quantum communication, and meditation science. 